\title{
Bracing - Halting Progression or Improving Curves in Adolescent Idiopathic Scoliosis
}

\author{
Wing-Yan CHAN ${ }^{1 *}$,Shu-Yan NG $^{2}$, Tsz-Ki HO ${ }^{1}$, Yin-Ling NG ${ }^{1}$ \\ ${ }^{1}$ Spine Technology Ltd, Wanchai, Hong Kong \\ ${ }^{2}$ Wanchai Chiropractic Clinic, Wanchai, Hong Kong
}

Received: August 18, 2016; Accepted: August 24, 2016; Published: August 2, 2016

*Corresponding author: Wing-Yan CHAN, Rm 1101, 11/fl Methodist House, 36 Hennessy Road, Wanchai, Hongkong, Tel: 852-25229673, Fax: 852-25273618; Email: wingyanc@ezped.com

\begin{abstract}
The effectiveness of spinal bracing in the treatment of adolescent idiopathic scoliosis has been controversial. Some studies have shown that bracing is only as effective as observation, whilst others have shown that bracing is superior to observation, halting progression and effectively reducing progression to surgical threshold. Recently, some studies have even shown improvement of curves with bracing. Yet, many of these studies have been judged to be of low methodological quality. In 2005, the Scoliosis Research Society (SRS) attempted to standardize the inclusion criteria and outcome measurements for bracing studies, to enable comparison among studies. In the guidelines, progression of $\leq 5^{\circ}$ is regarded as success. It is apparent that SRS did not regard improvement of curves probable. Improvement which is defined as a decrease of $\geq 6^{\circ}$ was not proposed until 2009. This may reflect an improvement in outcome with bracing in the last one to two decades.
\end{abstract}

The present review attempts to determine if there is a trend of improvement in outcome with bracing in the last 3 decades. Manual literature search was made in the PubMed using the keywords of brace, conservative treatment and adolescent idiopathic scoliosis. Relevant English articles on the outcome of wearing rigid braces from 1990-2016 were retrieved and reviewed to determine if there is a trend towards improvement in outcome with bracing and if bracing halts progression and improves curves.

Results show that there has been an apparent improvement in the effectiveness of bracing in reducing surgical rate since 2005 . Close inspection shows that the reduction in surgical rate is not due to an improvement over time, but is related to the types of brace. The effectiveness of Boston brace is not consistent over the years. The surgical rates vary and no consistent trend of improvement can be discerned in the last 2-3 decades. The surgical rate reported in 2007 for TLSO was as high at $79 \%$ and that in 2014 for Boston brace was 28\%. The surgical rates with European braces (Progressive Action Short Brace (PASB), Cheneau derivatives and Lyon/Sforzesco braces), however, are consistently lower, at less than $8 \%$. Similarly, the European braces have been found to be able to improve curves in over $50 \%$ of the at risk patients. Bracing does not therefore only halt progression of curves. Given a well-constructed brace, with good patient compliance, improvement of curves in over $50 \%$ of the patients is possible, particularly when used in conjunction with scoliosis specific exercises.

\section{Introduction}

Spinal bracing is at present a standard treatment in growing patients with adolescent idiopathic scoliosis with Cobb angle in excess of $20-25^{\circ}[1,2]$. Yet, systematic reviews regarded that the evidence in support of bracing in the treatment of Adolescent Idiopathic Scoliosis (AIS) is low [3, 4]. As late as 2007, Dolan et al reported that there was a high degree of variability in opinion among clinicians concerning the effectiveness of bracing. Some clinicians embraced the treatment, whilst others utterly rejected using braces for treatment [5].

A critical review of 15 studies on bracing and 3 studies on observation showed virtually no difference in the effectiveness of Thoracolumbosacral Orthosis (TLSO) bracing versus observation [6]. The pooled bracing surgical rate from the 18 studies was $23 \%$ compared with $22 \%$ in the observation group, showing no clear advantage of either approach[6].This view was similarly held by Goldberg et al (2001), who found that the incidence of surgery in their centre $(28.1 \%)$ did not differ significantly from another with aggressive orthotic policy (22.4\%). They questioned if bracing has any meaningful advantage [7].

Yet, many studies have shown that there is a decreased risk of curve progression with bracing as compared to observation $[8,9,10]$. Emans et al (1986) followed up 295 patients one year after weaning of the Boston brace [8]. They found that $49 \%$ of the curves had no change (remained $\pm 5^{\circ}$ of pre-brace angle). $7 \%$ of the patients worsened by $\geq 5^{\circ}$ and $11 \%$ of the patients received surgery [8]. Similarly, a prospective, multicenter study showed that bracing was more effective than observation and electrical stimulation [9]. At four years the successful rate, defined as progression of $\leq 6^{\circ}$ of bracing was $74 \%$, whereas those of observation and electrical stimulation were $34 \%$ and $33 \%$ respectively [9]. Yet, the study was regarded as of low methodological quality, as it was not randomized, non-blinded and the baseline differences between the groups were not statistically adjusted for and the results did not include the surgical rates $[3,6]$. Danielsson and colleagues (2007) followed a 
subset of Swedish patients from this study for 16 years, comparing the effectiveness of bracing versus observation in two groups of patients with comparable curves and demographics [10]. Results showed that in the braced group, no patient progressed by more than $\geq 6^{\circ}$. In the observation group, however, $40 \%$ of the patients progressed by $\geq 6^{\circ} ; 20 \%$ required brace treatment; and 6 patients (9.2\%) required surgery [10]. A multicenter study in 2013 which enrolled randomized cohort and preference cohort showed that TLSO effectively reduced the percentage of braced patients with curve progression to surgical threshold [11]. Indeed, the difference in outcome between bracing and not bracing was so significant that the trial was stopped early [11].

In view of the contradiction and the difficulties of comparing different studies, which used different inclusion and outcome criteria, Scoliosis Research Society (SRS) established a guideline for bracing studies to enable ease of comparison of effectiveness of braces in subsequent studies. Reviewing 32 bracing studies, Richards et al (2005) determined the inclusion criteria that would best identify patients most at risk of progression and the most appropriate definitions for bracing effectiveness [1]. It was determined that the patient braced should be 10 years or older, with curves between $25-40^{\circ}$ and Risser sign of $0-2$. If the patient is a female, she should be either premenarchal or less than one year postmenarchal. Assessment of brace effectiveness should include: the percentage of patients with $\leq 5^{\circ}$ curve progression, the percentage of patients with progression $\geq 6^{\circ}$ at skeletal maturity, the percentage of patients with curves exceeding the surgical threshold of $45^{\circ}$ at maturity and the percentage of patients who require surgical intervention during the 2-year follow up beyond maturity [1]. It is of note that the success of the bracing was defined as progression of $\leq 5^{\circ}$ [1]. Presumably, improvement of curves with bracing was deemed improbable and was not defined in the Scoliosis Research Society (SRS) guidelines [1].

Negrini et al (2009) defined improvement of curves as a reduction of $\geq 6^{\circ}$ at skeletal maturity when compared to prebrace angle [12]. This possibly stems from their findings that bracing can improve curves. Thus the successful rate as defined by SRS guidelines includes the improvement rate. To better define the terms, the present review regards change of curve $\pm 5^{\circ}$ as stabilization of curves. Successful rate is thus the sum of stabilization rate and improvement rate.

The SRS criteria did not take into consideration of the standards of bracing. The Society on Scoliosis Orthopedic and Rehabilitation Treatment (SOSORT) convened in 2008 and established a consensus concerning the standard of management of adolescent idiopathic scoliosis with bracing, with the aim to increase the efficacy and compliance to treatment [2]. SOSORT recommends clinicians to follow the guidelines in clinical practice [2].

Since the establishment of SRS and SOSORT guidelines [1, 2], there have been a number of studies. With uniformity of inclusion criteria and outcome measures, comparison of the effectiveness of bracing should theoretically become easier. We review the studies in the past 2-3 decades to determine if the effectiveness of bracing has improved in terms of halting curves progression, reducing the percentage of patients requiring surgical intervention and improving curves, particularly after the adoption of the two guidelines.

\section{Materials and Methods}

Studies from 1990 were manually searched in PubMed, using the key words "brace", "conservative treatment", "adolescent idiopathic scoliosis". Articles on early onset idiopathic scoliosis, secondary scoliosis and adult scoliosis were excluded. Also, articles on soft braces, night time braces and surgery were excluded. Studies using outcome measures other than Cobb angles were excluded. Only articles that were English or having English abstract were included. Studies that determined the combined effects of bracing and scoliosis specific exercises were included, whereas those dedicated solely to scoliosis specific exercises were excluded.

The articles were screened by the lead author for relevance. Only those articles that included inclusion criteria and outcome measures similar to that stipulated by the SRS guidelines were included for comparison. Studies that reported bracing for curves in excess of $40^{\circ}$ were not included. Data from studies published from 1990 onwards are reviewed to determine if the effectiveness of bracing has improved over the years, in terms of halting progression of curves, reducing surgical rates and improving curves in patients with AIS.

\section{Results}

The search revealed 596 papers. Basing on the above inclusion and exclusion criteria, 33 papers from 1990 - 2000, 53 papers from 2000 - 2010 and 42 papers from 2010 - 2016 were found. Papers that reported short term results of bracing and bracing designs were excluded. This leaves 53 studies for review.

The methodologies, inclusion criteria and outcome measurements of these studies differed significantly and quantitative comparison of the outcome measurements is impossible. Comparison is made qualitatively.

From 1990 to 2002, the reviewed English studies were braces designed in USA. Braces studied included the Milwaukee braces $[9,13,14,15]$, Wilmington braces $[16,17]$ and Boston or TLSO braces [18 - 23]. We were not aware of any English publication on the European braces during this time period. Since 2003, however, the number of publication on the effectiveness of European braces has increased dramatically [12, $24-31]$. This may be a result of the establishment of SOSORT in 2005.

\section{Progression to Surgical Threshold of $45^{\circ}$ and Surgical Rates}

From the data, it is evident (Figure 1) that the surgical rates reduce from the year 2005. Apart from the Janicki et al (2007) study which followed the SRS guidelines and reported $79 \%$ surgical rate [32], the surgical rates with bracing after 2005 hovered around 10\%. Close inspection showed that the reduction in surgical rates is not related to the improvement in 
effectiveness of a particular brace with time. The improvement is brace-dependent (Figure 2). Yet, brace with the same name can yield very dissimilar outcome. The percentage of patients requiring surgical intervention after wearing Wilmington brace varies from $13.4 \%$ [16] to $56 \%$ [17]. Also, the surgical rate of patients treated by Boston or TLSO brace varies from $0 \%[10,20]$ to over $60 \%$ for patients under the age of 13 and with curve in excess of $30^{\circ}[21]$.

Progression to surgical threshold was not reported in many studies. It similarly varies significantly from 3\% [14] to $46 \%$ [20, 33].

Interestingly, European braces which include the Lyon braces $[28,34]$, the Cheneau derivatives $[24,26,29,35]$, the Sibilla braces $[34,36]$ and the PASB $[27,37]$ are associated with a significant lower rate of surgery. The highest surgical rate reported was $7.9 \%$ [38], with some studies reporting no surgery after bracing $[12,29,31,36,37]$.

\section{Successful Rate (Progression $\leq 5^{\circ}$ )}

The successful rate, or the percentage of patients progressing $\leq 5^{\circ}$ is high for Wilmington brace $[16,17]$, Boston brace $[10,11$, $19,20,39,40]$ and Milwaukee brace [10, 14]. Danielsson et al (2007) and Weinstein et al (2013) reported that 100\% and 72\% of the braced patients did not progress more than $5^{\circ}$ respectively $[10,11]$.

For the European braces, the successful rate is high as well. The highest reported was 100\% [35 -37].

\section{Improvement Rate (Curve Reduction $\geq 6^{\circ}$ )}

Review of the papers showed that between 1990-2002, only 4 papers showed an improvement of curves of $\geq 6^{\circ}$ after bracing at skeletal maturity $[16,18,19,21]$. Piazza et al (1990) followed up 67 patients with AIS, wearing Wilmington brace [16]. For 42 patients with Cobb angles $20-29^{\circ}, 24 \%$ of them improved and for 34 patients with Cobb angles $30-39^{\circ}, 27 \%$ improved. The improvement was more marked in thoracic scoliosis $(29 \%)$, than double major curve (21\%) and thoracolumbar curve $(16 \%)$. Willers et al (1993) reported an improvement of curve in 3 out of the 25 patients $(12 \%)$ after weaning of Boston brace at skeletal maturity [18]. Similarly Goldberg et al (1995) reported an improvement of curve of more than $6^{\circ}$ in 3 (9.4\%) of the 32 patients, with mean age of 13.1 and Cobb angle of $22.2^{\circ}$ at skeletal maturity after wearing Boston brace [19]. Of interest is that the Cobb angle of three of the five patients (60\%) with lumbar curve improved. Fernandez-Feliberti et al (1995) followed up 75 female and 26 male AIS patients wearing TLSO until skeletal maturity [21]. They found a significant difference in the improvement rate in those with Cobb angle below and above $30^{\circ}$ and aged below and above age of 13. For patients below the mean age of 13, those with Cobb angle less than $29^{\circ}$ had a higher improvement rate of $54 \%$ as opposed to the $26 \%$ in patients with Cobb angle in excess of $30^{\circ}$. For patients with mean age above 13 , however, those with Cobb angle less than $29^{\circ}$ had a lower improvement rate of $58 \%$ as opposed to the $70 \%$ in patients with Cobb angle in excess of $30^{\circ}[21]$.

From 2003 onwards, we were able to identify 21 papers reporting an improvement of curves after weaning of braces at skeletal maturity $[24,25,26,29,31,38,42,43]$. The improvement rate varies from $24.2 \%$ for the Rigo Cheneau brace [30] to $94 \%$ for the Progressive Action Short Brace (PASB) (Figure 3) [27].

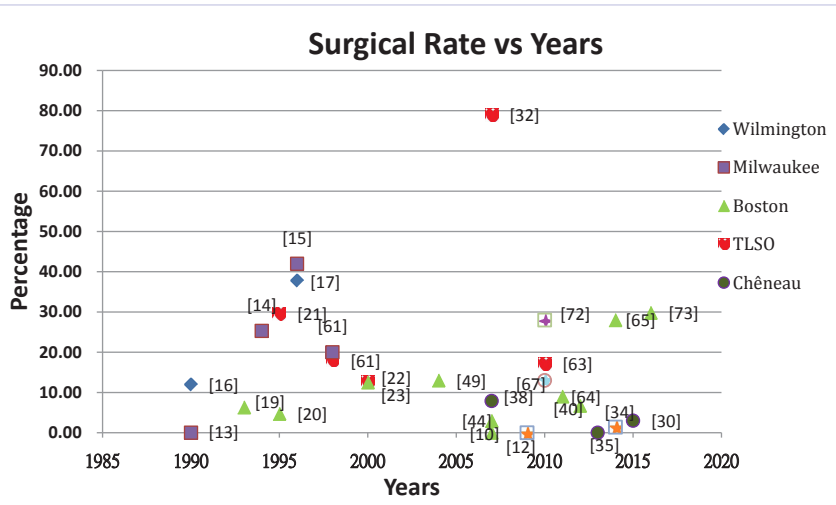

Figure 1: The surgical rates of bracing studies on AIS from 1990 to 2016.

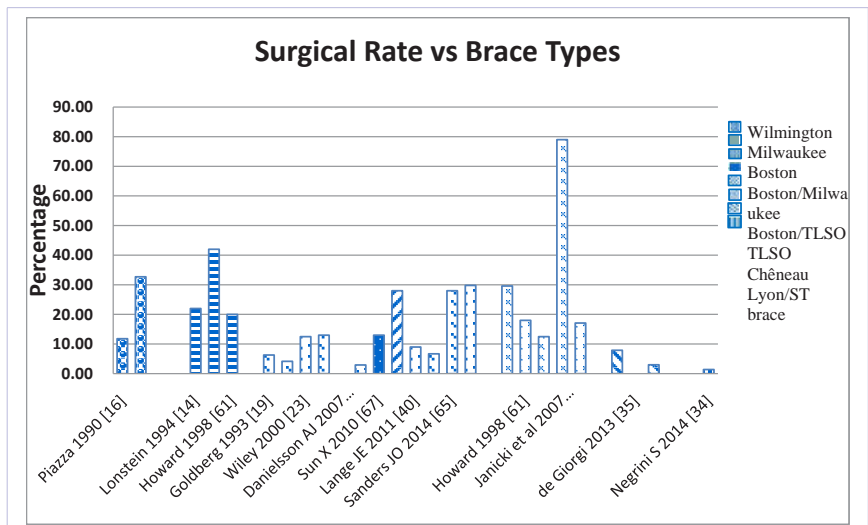

Figure 2: The surgical rates of different major braces in treating AIS reported from 1990 to 2016.

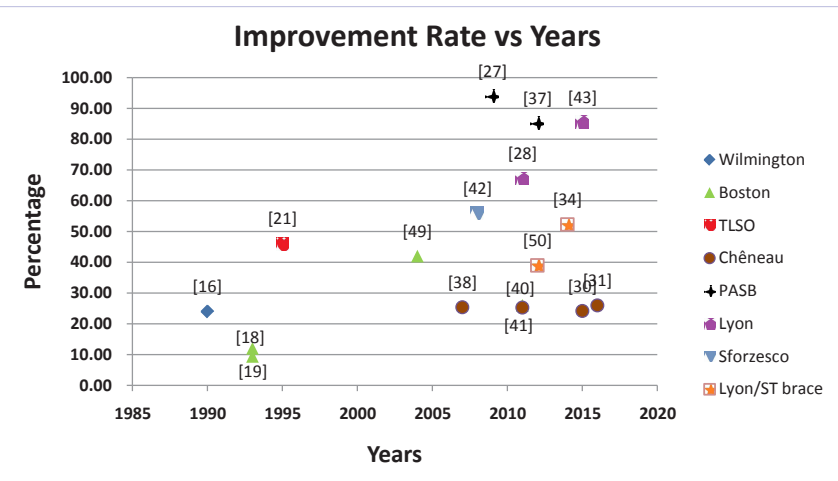

Figure 3: The improvement rates of bracing studies on AIS from 1990 to 2016 . 
It is to be noted, however, that PASB is specifically tailored for the treatment of thoracolumbar and lumbar curves, which are generally more responsive to bracing than thoracic scoliosis and double major curves.

\section{Stabilization Rate (Change of Curve $\pm 5^{\circ}$ )}

When the successful rate (progression $\leq 5^{\circ}$ ) is further divided into stabilization rate (change of curve $\pm 5^{\circ}$ ) and improvement rate (curve reduction $\geq 6^{\circ}$ ), it is easier to observe the effectiveness of bracing. Lange et al (2011)found that the successful rate of Boston brace was $88.5 \%$ [40], while de Mauroy et al (2011) reported the successful rate of Lyon brace at 95\% [28]. These two figures give an impression that both brace types are similar in effectiveness. Comparison of their improvement rates and the stabilization rates, however, show difference of effectiveness. The improvement and stabilization rates of Lyon brace study by de Mauroy (2011) was $67.2 \%$ and $27.80 \%$ respectively [28], whereas the improvement and stabilization rates of Boston brace reported by Lange et al (2011) was $26 \%$ and $62.5 \%$ respectively [40]. The figures infer that the Lyon brace is more effective than the Boston brace in improving curves in treatment of AIS.

\section{Discussion}

Bracing has been used in the treatment of AIS since the early $50 \mathrm{~s}$, with the primary objective of halting progression. Bracing is not regarded as being able to improve curves. It is possibly on this background that SRS regarded a progression of $\leq 5^{\circ}$ as a success [1]. Interestingly, however, after Negrini et al (2009) defined improvement of curves as a reduction of $\geq 6^{\circ}$, many studies have since reported improvement of curves with bracing [12].

When comparing the percentage of patients progressing to surgical threshold, the surgical rates and the improvement rate in the past 26 years, we find that there is a trend towards reduction in surgical rate and an increase in improvement rate. Yet, close inspection showed that the change is more related to the type of brace used, rather than an improvement in the effectiveness of a particular type of brace over time.

\section{Progression to Surgical Threshold of $45^{\circ}$ and Surgical Rate}

Comparing the data in the last 3 decades, we find there is an apparent trend towards reduction in surgical rate (Figure 1). However, close inspection showed that the data points refer to different braces. The reduction in surgical rates is found to be related to the types of brace, rather than an improvement of a particular brace over time. Also we find that the percentage of patients progressing to surgical threshold and the surgical rate differ significantly among different braces. The surgical rates of patients treated by Boston braces and TLSO over the time vary considerably, from $0 \%$ [10] to $79 \%$ [32]. The surgical rates of patients treated with European braces (PASB, Lyon and Sforzesco braces) are consistently lower.

The surgical rates reported for the Boston braces varied from less than $10 \%[19,20,44]$ to above $30 \%[15,17,21]$. Some of the more recent reports had surgical rates as high as $60-70 \%$
$[32,45]$. The reasons for the high failure rate were not explained. It is possible that the high failure rate in the Spoonamore et al (2004) [45] study was related to the use of Rosenberger brace, which was still in the developmental stage[45] and the Janicki et al (2007)study, which was the first study that followed the SRS guidelines, involved patients at risk of progression [32]. Yet, not all the TLSO had as high a failure rate. Kessler et al (2008) reported a much lower percentage of patients progressing to surgical threshold, using the Los Angeles brace. The surgical rate reported was only $5 \%$ [46].

The authors are unable to identify any English publication before 2003 using the European braces for comparison. Most of the publications in English on European braces were after 2003. Rigo et al (2003) reported the effectiveness of Rigo Cheneau brace in the treatment of AIS [26]. In the worst case analysis, assuming that patients who were lost to follow up progressed to surgical threshold, the percentage of patients progressing to surgical threshold was $14.1 \%$, which was far below that reported for Boston braces[47] and other TLSO[21]. For the Cheneau derivatives, the surgical rates reported ranged from $0[29,31,35]$ to less than $10 \%[30,38]$. Zaborowska et al reported a surgical rate of $12.7 \%$ [41]. The percentage of patients progressing to surgical threshold was similar in the study by Maruyama et al (2015)and Rigo et al (2003) at $12.1 \%$ and $14.1 \%$ respectively $[26,30]$.

The PASB brace which was fabricated specifically to treat thoracolumbar and lumbar curves had the best results, with surgical rates and percentage of patients progressing to surgical threshold of $45^{\circ}$ reported to be $0[27,37]$.

The Italian Isico group employed different braces, which included the Sibilla brace, Lyon brace, Sforzesco brace and ART brace basing on individual patient needs also achieved good results $[34,36]$. Only $1.4 \%$ of the patients progressed to surgical threshold in one study [34]. In another study, the percentage was 0 [36]. It has, however, to be noted that in the studies, scoliosis specific exercises were used in conjunction with bracing. Courvoiser et al (2013) using a combination of Cheneau brace, Lyon brace, Milwaukee and Charleston braces also reported zero percentage of patients progressing to surgical threshold [48].

\section{Improvement in Curves $\left(\geq 6^{\circ}\right)$}

Successful treatment was defined as progression of $\leq 5^{\circ}[1]$, indirectly suggesting that bracing would not improve curves. The number of studies reporting improvement rate was few before Negrini et al (2009) defined the improvement rate [12]. After 2009 , the number of studies reporting improvement of curves increase dramatically (Figure 3).

For Boston brace, Piazza etal (1990) reported an improvement rate of $21 \%$ [16]. Goldberg reported an improvement of $60 \%$ in the three lumbar patients. Fernandez-Feliberti et al (1995) reported $70 \%$ improvement rate in patients over the age 13 and with Cobb angle in excess $30^{\circ}[21]$. Lange et al (2011) and Vijverman et al (2004) reported an improvement rate of $26 \%$ and $42 \%$ respectively $[40,49]$. 
Similar improvement rates were reported for Cheneau derivatives. The reported improvement rates ranged from around $25 \%[30,38,41]$ to $30 \%[24,31]$.Landauer et al $(2003)$ reported a successful rate of $78 \%$, using Cheneau brace. However, they did not report the improvement rate [24].

The improvement rate of Progressive Action Short Brace (PASB) is more significant. The reported improvement rate was above $85 \%$ [37] and was as high as $94 \%$ [27]. The improvement rate of Lyon brace was also significant at $85.5 \%$ [43]. The improvement rate in using a combination of braces basing on individual needs was also good $[34,50,51]$, ranging from $39 \%$ [50] to $65.7 \%$ [51].

\section{Halting Progression or Improving Curves}

From the data, it is apparent that the effectiveness of bracing hinges on the type, quality and fitting of braces (Figure 4). The compliance of the patients and incorporation of the scoliosis specific exercises may improve the outcome of bracing.

The lower percentage of patients reaching surgical threshold and the surgical rates in patients wearing European braces as compared to those wearing Boston braces may be related to the brace design and the treatment approach adopted by physicians using the braces.

Boston braces are generally employed by orthopedic surgeons. The objective of the brace is to halt progression of curves to below surgical threshold. In case the brace fails to achieve the objective, the patient can be treated surgically. On the contrary, the European braces are predominantly used by physicians who treat patients conservatively. Failure of the brace would require them to refer the patients for surgical treatment. They are motivated to improve the outcome of the brace treatment.

Boston brace was designed to have zero lumbar lordosis [20], as the effectiveness partially relies on the reduction of lumbar lordosis [20]. Yet, van Loon et al (2008) have shown that forced thoracolumbar lordosis would reduce coronal curves in patients with double major scoliosis [52]. Also, Boston braces encourage thoracic flat back, which has been shown to be detrimental to the correction of curves [53].

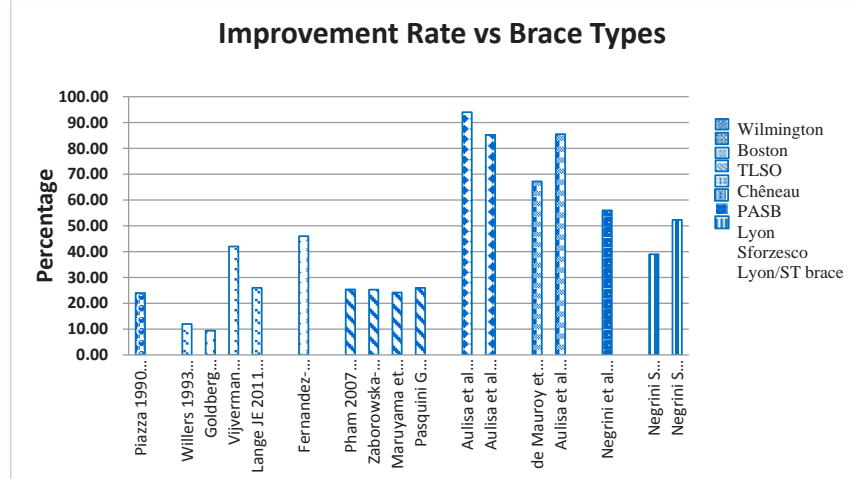

Figure 4: The improvement rates of different major braces in treating AIS reported from 1990 to 2016.
The effectiveness of a brace also depends on how it is made. The quality of the brace cannot be judged by its name [54]. Braces with the same name can produce markedly different results [54]. Danielsson et al (2007) pointed out the importance of the skill and dedication of the orthotist in the effectiveness of a brace [10]. They regarded the well dedicated orthotist as a bias or weakness of their brace study [10]. The orthotist was very committed to the bracing of scoliosis patients and by following the rules for performing brace treatment meticulously, he was able to obtain extraordinary good results [10]. Similar view is held by other authors [54,55]. Rigo et al $(2010)$ pointed out the importance of quality of the brace in the management of AIS [54]. With experience, the correction of the brace improved [25].

Apart from the design of the braces, the amount of in-brace correction and comfort are also important in the outcome. Large in-brace correction in excess of $50 \%$ would be accompanied by improvement at skeletal maturity $[24,56]$ and hours of brace wear are positively associated with the rate of treatment success $[11,17,21,23,24,57]$. Well design braces that are comfortable to wear may improve compliance, which is important in improvement of the outcome. Wearing a brace full time $(\geq 18$ hours) is associated with a much lower surgical rate than wearing the brace part time ( $\leq 12$ hours) [23].

From the review, it is noteworthy that the PASB produces remarkable improvement in thoracolumbar and lumbar curves $[27,37]$. No patient progressed $\geq 6^{\circ}$ and all the curves were either stabilized or improved [27, 37].

Also, bracing should not be employed alone in the management of AIS. Scoliosis specific exercises should be incorporated. The exercises improve the muscle strength of the trunk and the postural awareness of the patients. More importantly perhaps, they improved the curves $[58,59]$, reducing the loss of correction which frequently accompanied the weaning of brace $[14,15$, $18,19]$. A recent prospective study based on SRS and SOSORT criteria showed that with a combination of brace and scoliosis specific exercises, $52.3 \%$ of the 73 patients improved [34].

Recently, it was reported that bracing can help reduce curves in excess of $45^{\circ}$ [60]. It is thus timely to discuss if the objective of the brace remains to halt progression of curves, or to improve the curves and if the indications for bracing should be broadened to include patients with curves around the surgical threshold.

\section{Limitation and Future Directions}

The main weakness of the present study is that it is a qualitative review, comparing studies with different patient demographics, curve patterns, severity of curves, braces, bracing strategies and outcome measures. Many studies used TLSO, without defining the type of braces fabricated. Also, some studies used a combination of braces, making comparison among braces very difficult. PASB is a rigid brace specifically made to correct thoracolumbar and lumbar curves, which are easier to correct than thoracic and double major curves. Direct comparison of its effectiveness with that of other braces may not be appropriate. 
Another limitation of the present review is that the surgical rates instead of the percentage of patients progressing to surgical threshold are compared. Some patients may progress to surgical threshold and yet refuse to receive surgery, especially when they are taken care of by physicians who opt for conservative treatment. Thus comparing the surgical rates may be somewhat misleading. No comparison of the percentage of patients progressing to surgical threshold was made, as only a few papers contain such data. Also many studies reported successful rate, but did not differentiate between improvement rate and stabilization rate.

To enable better comparison, future bracing studies should adopt the SRS and SOSORT guidelines. Patient population should preferably be more homogeneous, grouping by curve types as well. The in-brace correction should be reported as an assurance of the quality of brace. The compliance of the brace wearing should also be recorded. The dosage and frequency of scoliosis specific exercises, if employed should be reported. Study should also record the improvement rate, the stabilization rate, progression rate and the percentage of patients progressing to surgical threshold.

\section{Conclusion}

Bracing does not only halt progression of curves. Given a wellconstructed brace, with good patient compliance, improvement of curves in some patients can be expected. The improvement rate can possibly be increased by the simultaneous incorporation of scoliosis specific exercises.

\section{Conflict of Interest}

All the authors declare no conflict of interest.

\section{References}

1. Richards BS, Bernstein RM, D'Amato CR, Thompson GH. Standardization of criteria for adolescent idiopathic scoliosis brace studies: SRS Committee on bracing and nonoperative management. Spine (Phila Pa 1976). 2005;30(18):2068-2075.

2. Negrini S, Grivas TB, Kotwicki T, Rigo M, Zaina F. International Society on ScoliosisOrthopaedic and Rehabilitation Treatment (SOSORT) Guidelines on "Standards of management of idiopathic scoliosis with corrective braces in everyday clinics and in clinical research": SOSORT Consensus 2008. Scoliosis. 2009;4:2. doi:10.1186/1748-7161-4-2.

3. Negrini S, Minozzi S, Bettany-Saltikov J, Zaina F, Chockalingam N, Grivas TB, Kotwicki T et al. Braces for idiopathic scoliosis in adolescents. Cochrane Database Syst Rev. 2010;(1):CD006850. doi: $10.1002 / 14651858$.

4. Negrini S, Minozzi S, Bettany-Saltikov J, Chockalingam N, Grivas TB, Kotwicki $\quad T$ et al. Braces for idiopathic scoliosis in adolescents. Cochrane Database Syst Rev. 2015;(6):CD006850.

5. Dolan LA, Donnelly MJ, Spratt KF, Weinstein SL. Professional opinion concerning the effectiveness of bracing relative to observation in adolescent idiopathic scoliosis. J Pediatr Orthop. 2007 AprMay;27(3):270-276. D0I:10.1097/01.bpb.0000248579.11864.47.

6. Dolan LA, Weinstein S. Surgical rates after observation and bracing for adolescent idiopathic scoliosis: an evidence-based review. Spine 2007;32(19):S91-S100. DOI:10.1097/BRS.0b013e318134ead9.

7. Goldberg CJ, Moore DP, Fogarty EE, Dowling FE. Adolescent idiopathic scoliosis - the effect of brace treatment on the incidence of surgery. Spine. 2001;26(1):42-47.
8. Emans JB, Kaelin A, Bancel P, Hall JE, Miller ME. The Boston bracing system for idiopathic scoliosis. Follow up results in 295 patients. Spine. 1986;11(8):792-801.

9. Nachemson AL, Peterson LE. Effectiveness of treatment with a brace in girls who have adolescent idiopathic scoliosis. A prospective, controlled study based on data from the Brace Study of the Scoliosis Research Society. J Bone Joint Surg Am. 1995;77(6):815-822.

10. Danielsson AJ, Hasserius R, Ohlin A, Nachemson AL. A prospective study of brace treatment versus observation alone in adolescent idiopathic scoliosis. A follow-up mean of 16 years after maturity. Spine 2007;32(20):2198-2207. DOI:10.1097/BRS.0b013e31814b851f.

11. Weinstein SL, Dolan LA, Wright JG, Dobbs MB. Effects of bracing in adolescents with idiopathic scoliosis. The New Engl J Med. 2013;369(16):1512-1521.

12. Negrini S, Atanasio S, Fusco C, Zaina F. Effectiveness of complete conservativetreatment for adolescent idiopathic scoliosis (bracing and exercises) based onSOSORT management criteria: results according to the SRS criteria for bracing studies - SOSORT Award 2009 Winner. Scoliosis. 2009;4:19. DOI:10.1186/1748-7161-4-19.

13. Montgomery F, Willner S, Applegren G. Long-term follow-up of patients withadolescent idiopathic scoliosis treated conservatively: An analysis of the clinical value of progression. J Pediatr Orthop. 1990;10(1):48-52.

14. Lonstein JE, Winter RB.The Milwaukee brace for the treatment of adolescent idiopathic scoliosis. A review of one thousand and twenty patients. J Bone Joint Surg Am.1994;76(8):1207-1221.

15. Noonan KJ, Weinstein SL, Jacobson WC, Dolan LA. Use of the Milwaukee brace for progressive idiopathic scoliosis. J Bone Joint Surg Am. 1996;78(4):557-567.

16. Piazza MR, Bassett GS. Curve progression after treatment with the Wilmington Brace for idiopathic scoliosis. J Pediatr Orthop. 1990;10(1):39-43.

17. Allington NJ, Bowen JR, Delaware W. Adolescent idiopathic scoliosis: treatment with the Wilmington brace. J Bone Jt Surg Am. 1996; 78(7):1056-1062.

18. Willers U, Normelli H, Aaro S, Svensson O, Hedlund R. Long-term results of Boston brace treatment on vertebral rotation in idiopathic scoliosis. Spine (Phila Pa 1976). 1993;18(4):432-435.

19. Goldberg CJ, Dowling FE, Hall JE, Emans JB. A statistical comparison between natural history of idiopathic scoliosis and brace treatment in skeletally immature adolescent girls. Spine. 1993;18(7):902-9088.

20. Olafsson Y, Saraste H, Soderlund V, Hoffsten M. Boston brace in the treatment of idiopathic scoliosis. J Pediatr Orthop. 1995;15(4):524527.

21. Fernandez-Feliberti R, Flynn J, Ramirez N, Trautmann M, Alegria M. Effectiveness of TLSO bracing in the conservative treatment of idiopathic scoliosis. J Pediatr Orthopedics. 1995;15(2):176-181.

22. Korovessis P, Kyrkos C, Piperos G, Soucacos PN. Effects of thoracolumbosacralorthosis on spinal deformities, trunk asymmetry, and frontal lower rib cage in adolescent idiopathic scoliosis. Spine (Phila Pa 1976). 2000;25(26):2064-2071.

23. Wiley JW, Thomson JD, Mitchell TM, Smith BG, Banta JV. Effectiveness of the Boston brace in treatment of large curves adolescent idiopathic scoliosis. Spine. 2000;25(18):2326-2332.

24. Landauer F, Wimmer C, Behensky H. Estimating the final outcome of brace treatment for idiopathic thoracic scoliosis at 6-month follow-up. 
Pediatr Rehabil. 2003;6(3-4):201-207. DOI:10.1080/1363849031000 1636817.

25. Grivas TB, Vasiliadis E, Chatziargiropoulos T, Polyzois VD, Gatos K The effect of a modified Boston brace with anti-rotatory blades on the progression of curves in idiopathic scoliosis: aetiologic implications. Pediatric Rehabil. 2003;6(3-4):237-242. DOI.10.1080/13638490310 001636808.

26. Rigo MD, Reiter $\mathrm{CH}$, Weiss HR. Effect of conservative management on the prevalence of surgery in patients with adolescent idiopathic scoliosis. Pediatric Rehabil. 2003;6(3-4): 209-214. DOI:10.1080/136 38490310001642054

27. Aulisa AG, Guzzanti V, Galli M, Perisano C, Falciglia F, Aulisa L Treatment ofthoraco-lumbar curves in adolescent females affected by idiopathic scoliosis with a Progressive Action Short Brace (PASB): assessment of results according to the SRS committee on bracing and non operative management standardization criteria. Scoliosis 2009;4:21. Doi:10.1186/1748-7161-4-21.

28. de Mauroy JC, LecanteC, Barral F. The Lyon approach to the conservative treatment of scoliosis. Scoliosis. 2011;6:4. doi:10.1186/1748-7161-6-

29. Weiss HR, Werkmann M. Rate of surgery in a sample of patients fulfilling the SRS inclusion criteria treated with a Chêneau Brace of actual standard. Stud Health Technol Inform. 2012;176:407-410.

30. Maruyama T, Kobayashi Y, Miura M, Nakao Y. Effectiveness of brace treatment for adolescent idiopathic scoliosis. Scoliosis 2015;10(2):S12. DOI: 10.1186/1748-7161-10-S2-S12

31. Pasquini G, Cecchi F, Bini C, MolinooLova R, Vannetti F, Castagnoli C, Paperini A et al. The outcome of modified version of the Cheneau brace in Adolescent Idiopathic Scoliosis (AIS) based on SRS and SOSORT criteria: a retrospective study. Eur J Phys Rehabil Med. 2016;4.

32. Janicki JA, Poe-Cohert C, Armstrong DG, Thompson GH. A comparison of the thoracolumbosacral orthoses and Providence orthoses in the treatment of adolescent idiopathic scoliosis. J Pediatr Orthop. 2007; 27(4):369-374. DOI:10.1097/01.bpb.0000271331.71857.9a.

33. Karol LA. Effectiveness of bracing in male patients with idiopathic scoliosis. Spine (PhilaPa 1976). 2001 Sep 15;26(18):2001-2005.

34. Negrini S, Donzeli S, Lusini M, Minnella S, Zaina F. The effectiveness of combined bracing and exercise in adolescent idiopathic scoliosis based on SRS and SOSORT criteria: a prospective study. BMC Musculoskeletal Discord. 2014;15:263. DOI:10.1186/1471-2474-15263.

35. De Giorgi S, Piazzolla A, Tafuri S, Borracci C, Martucci A, De Giorgi G. Chêneau brace for adolescent idiopathic scoliosis: long-term results. Can it prevent surgery? Eur Spine J.2013;22(6):S815-22. doi:10.1007/ s00586-013-3020-1.

36.Zaina F, de Mauroy JC, Donzelli S, Negrini S. A multicenter study comparing the SPoRT and ART braces effectiveness according to the SOSORT-SRS recommendations. Scoliosis. 2015;10:23. DOI:10.1186/ s13013-015-0049-4

37. Aulisa AG, Guzzanti V, Perisano C, Marzetti E, Falciglia F, Aulisa L.Treatment of lumbar curves in scoliotic adolescent females with progressive action short brace: a case series based on the Scoliosis Research Society Committee Criteria. Spine (Phila Pa 1976). 2012;37(13):E786-E791. doi:10.1097/BRS.0b013e31824b547d.

38. Pham VM, Herbauxb B, Schilla A, Thevenon A. Evaluation of the Chêneau brace inadolescent idiopathic scoliosis. Annales de réadaptation et de MédecinePhysique. 2007;50(3):125-133. DOI:10.1016/j.annrmp.2006.11.003.
39. Lou E, Hill D, Raso J, Mahood J, Moreau M. Prediction of brace treatment outcomes by monitoring brace usage. Stud Health Technol Inform 2006:123:239-244.

40. Lange JE, Steen H, Gunderson R, Brox JI. Long-term results after Boston bracetreatment in late-onset juvenile and adolescent idiopathic scoliosis. Scoliosis. 2011;6:18. doi:10.1186/1748-7161-6-18.

41.Zaborowska-Sapeta K, Kowalski IM, Kotwicki T, ProtasiewiczFałdowska H, Kiebzak W. Effectiveness of Chêneau brace treatment for idiopathic scoliosis: prospective study in 79 patients followed to skeletal maturity. Scoliosis. 2011;6(1):2. doi:10.1186/1748-7161-6-2.

42. Negrini S, Atanasio S, Negrini F, Zaina F, Marchini G. The Sforzesco brace can replace cast in the correction of adolescent idiopathic scoliosis: A Controlled prospective cohort study. Scoliosis. 2008;3:15. doi:10.1186/1748-7161-3-15.

43. Aulisa AG, Guzzanti V, Falciglia F, Giordano M, Marzetti E, Aulisa L. Lyon bracing in adolescent females with thoracic idiopathic scoliosis: a prospective study based on SRS and SOSORT criteria. BMC Musculoskelet Disord. 2015;16:316. doi:10.1186/s12891-015-0782-

44. Yrjonen T, Ylikoski M, Schlenzka D, Poussa M. Results of brace treatment ofadolescent idiopathic scoliosis in boys compared with girls: a retrospective study of 102 patients treated with the Boston brace. Eur Spine J. 2007;16:393-397. DOI:10.1007/s00586-0060167-z.

45. Spoonamore MJ, Dolan LA, Weinstein SL. Use of the Rosenberger brace in the treatment of progressive adolescent idiopathic scoliosis. Spine (Phila Pa 1976). 2004;29(13):1458-1464.

46. Kessler J. Efficacy of a new computer-aided design/computer aided manufacture orthoses in the treatment of adolescent idiopathic scoliosis. J Pediatr Orthop 2008; B17:207-211. doi.10.1097/ BPB.0b013e3283046117.

47. Watts HG, Hall JE, Stanish W. The Boston brace system for the treatment of low thoracic and lumbar scoliosis by the use of a girdle without superstructure. Clin Orthop. 1977;126:87-92.

48. Courvoisier A, Drevelle X, Vialle R, Dubousset J, Skalli W. 3D analysis of brace treatment in idiopathic scoliosis. Eur Spine J. 2013;22(11):24567. doi:10.1007/s00586-013-2881-7.

49. Vijvermans V, Fabry G, Nijs J. Factors determining the final outcome of treatment of idiopathic scoliosis with the Boston brace: a longitudinal study. J Pediatr Orthop B. 2004;(3):143-149.

50. Negrini S, Donzelli S, Lusini M, Zaina F. Bracing can reduce high degree curves andimprove aesthetics immediately after the end of growth. Final results of retrospective case series. Stud Health Technol Inform. 2012;176:393-396

51.Aulisa AG, Giordano M, Falciglia F, Marzetti E, Poscia A, Guzzanti V.Correlation between compliance and brace treatment in juvenile and adolescent idiopathic scoliosis: SOSORT 2014 award winner. Scoliosis. 2014;9:6. doi: 10.1186/1748-7161-9-6.

52.Van Loon PJ, Kühbauch BA, Thunnissen FB. Forced lordosis on the thoracolumbarjunction can correct coronal plane deformity in adolescents with double major curve pattern idiopathic scoliosis. Spine. 2008;1;33(7):797-801.doi:10.1097/BRS.0b013e3181694ff5.

53. Wynne JH. The Boston brace and TriaC system. Disabil Rehabil Assist Technol2008; 3(3):130-135. doi:10.1080/17483100801903988.

54. Rigo MD, Villagrasa M, Gallo. A specific scoliosis classification correlating with brace treatment: description and reliability. Scoliosis. 2010;5(1):1. doi:10.1186/1748-7161-5-1. 
55. Bowman GG. The L.A. brace-description and discussion. Physiotherapy Theory and Practice 2011;27(1):68-73. doi:10.3109/09593980903498352.

56. Appelgren G, Willner S. End Vertebra Angle - A roentgenographic method to describe a scoliosis. A follow-up study of idiopathic scoliosis treated with the Boston brace. Spine (Phila Pa 1976) 1990;15(2):7174.

57. Rowe DE, Bernstein SM, Riddick MF, Adler F, Emans JB, GardnerBonneau D. Ameta-analysis of the efficacy of non-operative treatments for idiopathic scoliosis. J Bone Joint Surg Am. 1997;79(5):664-674.

58. Monticone M, Ambrosini E, Cazzaniga D, Rocca B, Ferrante S. Active self-correctionand task-oriented exercises reduce spinal deformity and improve quality of life insubjects with mild adolescent idiopathic scoliosis. Results of a randomized controlled trial. Eur Spine J. 2014;23(6):1204-14. doi:10.1007/s00586-014-3241-y.

59.

60. Kuru T, Yeldan İ, Dereli EE, Özdinçler AR, Dikici F, Çolak İ. The efficacy of three-dimensional Schroth exercises in adolescent idiopathic scoliosis: a randomised controlled clinical trial.Clin Rehabil. 2016 Feb;30(2):181-90. doi:10.1177/0269215515575745.

61. Lusini M, Donzelli S, Minnella S, Zaina F, Negrini S. Brace treatment is effective in idiopathic scoliosis over $45^{\circ}$ : an observational prospective cohort controlled study. Spine J. 2014; 22(11):2456-7. doi:10.1016/j. spinee.2013.11.040.

62. Howard A, Wright JG, Hedden D. A comparative study of TLSO, Charleston, and Milwaukee braces for idiopathic scoliosis. Spine (Phila Pa 1976). 1998;23(22):2404-2411.

63. Lange JE, Streen H, Brox JI. Long-term results after Boston brace treatment in adolescent idiopathic scoliosis. Scoliosis. 2009;4:17. DOI: $10.1186 / 1748-7161-4-17$.

64. Gammon SR, Mehlman CT, Chan W, Heifetz J, Durrett G, Wall EJ. A comparison of thoracolumbosacral orthoses and SpineCor treatment of adolescent idiopathic scoliosis patients using the Scoliosis Research
Society standardized criteria. J Pediatr Orthop. 2010;30(6): 531-8. doi:10.1097/BP0.0b013e3181e4f761.

65. Brox JI, Lange JE, Gunderson RB, Steen H. Good brace compliance reduced curve progression and surgical rates in patients with idiopathic scoliosis. Eur Spine J. 2012;2(10):1957-1963. doi: 10.1007/ s00586-012-2386-9.

66. Sanders JO, Newton PO, Browne RH, Katz DE, Birch JG, Herring JA. Bracing for idiopathic scoliosis: how many patients require treatment to prevent one surgery? J Bone Joint Surg Am. 2014;96(8):649-653. doi:10.2106/JBJS.M.00290.

67. Maruyama T, Kitagawa T, Takeshita K, Mochizuki K, Nakamura K. Conservative treatment for adolescent idiopathic scoliosis: can it reduce the incidence of surgical treatment? Pediatr Rehabil. 2003;6(34):215-219. DOI:10.1080/13638490310001642748.

68. Sun X, Wang B, Qiu Y, Zhu ZZ, Zhu F, Yu Y, Qian BP et al. Outcomes and predictors of brace treatment for girls with adolescent idiopathic scoliosis. Orthop Surg. 2010;2(4):285-290. doi:10.1111/j.17577861.2010.00101.x.

69. Ovadia D, Eylon S, Mashiah A, Wientroub S, Lebel ED. Factors associated with thesuccess of the RigoSystem Cheneau brace in treating mild to moderateadolescent idiopathic scoliosis. J Child Orthop. 2012;6(4):327-331. doi:10.1007/s11832-012-0429-8.

70. Negrini S, Marchini G. Efficacy of the symmetric, patient-oriented, rigid, threedimensional, active (SPoRT) concept of bracing for scoliosis: a prospective study of the Sforzesco versus Lyon brace. Eura Medicophys. 2007; 43(2):171-81.

71. Negrini S, Marchini G, Tomaello L. The Sforzesco Brace and SPoRT Concept (Symmetric, Patient-oriented, Rigid, Three-dimensional) versus the Lyon Braceand3-Point Systems for Bracing Idiopathic Scoliosis. Stud Health TechnolInform. 2006;176:245-249.

72. Bulthuis GJ, Veldhuizen AG, Nijenbanning G. Clinical effect of continuous corrective force delivery in the non-operative treatment of idiopathic scoliosis: a prospective cohort study of the Triac-brace. Eur Spine J. 2008;17(2):231-239. doi:10.1007/s00586-007-0513-9 